\title{
Uniform stability of fractional neutral systems: a Lyapunov-Krasovskii functional approach
}

KeWei Liu ${ }^{1,2^{*}}$ and Wei Jiang ${ }^{1}$

"Correspondence: kweiliu@163.com 'School of Mathematical Sciences, Anhui University, Hefei, 230039, China

${ }^{2}$ School of Mathematics, Hefei University of Technology, Hefei, 230009, China

\begin{abstract}
In this paper, we study the stability of nonlinear fractional neutral systems equipped with the Caputo derivative. We extend the Lyapunov-Krasovskii approach for the nonlinear fractional neutral systems. Conditions of uniform stability are obtained for the nonlinear fractional neutral systems.

MSC: $34 \mathrm{~K} 20 ; 34 \mathrm{~K} 37 ; 34 \mathrm{~K} 40$

Keywords: fractional neutral systems; uniform stability; Lyapunov-Krasovskii approach
\end{abstract}

\section{Introduction}

In recent decades, fractional calculus and fractional differential equations have attracted great attention. It has been proved that fractional calculus and fractional differential equations are valuable tools in the modeling of many phenomena in various fields of engineering, physics and economics. For details and examples, see [1-5] and the references therein.

Stability analysis is always one of the most important issues in the theory of differential equations and their applications for both deterministic and stochastic cases. The analysis on stability of fractional differential equations is more complex than that of classical differential equations, since fractional derivatives are nonlocal and have weakly singular kernels. Recently, stability of fractional differential equations has attracted increasing interest. The earliest study on stability of fractional differential equations started in [6], the author studied the case of linear fractional differential equations with Caputo derivative and the same fractional order $\alpha$, where $0<\alpha \leq 1$. The stability problem comes down to the eigenvalue problem of system matrix. Since then, many researchers have done further studies on the stability of linear fractional differential systems [7-11]. For the nonlinear fractional differential systems, the stability analysis is much more difficult and a few results are available in [12-18]. For more details about the stability results and the methods available to analyze the stability of fractional differential equations, the reader may refer to the recent survey papers $[19,20]$ and the references therein.

As we all know, Lyapunov's second method provides a way to analyze the stability of a system without explicitly solving the differential equations. It is necessary to extend Lyapunov's second method to fractional systems. In $[12,13]$, the fractional Lyapunov's second method was proposed, and the authors extended the exponential stability of integer order differential system to the Mittag-Leffler stability of fractional differential system. In [14], by using Bihari's and Bellman-Gronwall's inequality, an extension of Lyapunov's

\section{基 Springer}

(O2013 Liu and Jiang; licensee Springer. This is an Open Access article distributed under the terms of the Creative Commons Attribution License (http://creativecommons.org/licenses/by/2.0), which permits unrestricted use, distribution, and reproduction in any medium, provided the original work is properly cited. 
second method for fractional-order systems was proposed. In [15-17], Baleanu et al. extended Lyapunov's method to fractional functional differential systems and developed the Lyapunov-Krasovskii stability theorem, Lyapunov-Razumikhin stability theorem and Mittag-Leffler stability theorem for fractional functional differential systems. As far as we know, there are few papers with respect to the stability of fractional neutral systems. In this paper, we consider the stability of a class of nonlinear fractional neutral functional differential equations with the Caputo derivative. Motivated by Li et al. [12, 13], Baleanu et al. [15] and Cruz and Hale [21], we aim in this paper to extend the Lyapunov-Krasovskii method for the nonlinear fractional neutral systems.

The rest of the paper is organized as follows. In Section 2, we give some notations and recall some concepts and preparation results. In Section 3, we extend the LyapunovKrasovskii approach for the nonlinear fractional neutral systems, results of uniform stability for the nonlinear fractional neutral systems are presented. Conclusions are presented in Section 4.

\section{Preliminaries}

In this section, we introduce notations, definitions, and preliminary facts needed here. Throughout this paper, let $\mathbb{R}^{n}$ be a real $n$-dimensional linear vector space with the norm $|\cdot|$, let $\mathcal{C}=C\left([-r, 0], \mathbb{R}^{n}\right)$ be the space of continuous functions taking $[-r, 0]$ into $\mathbb{R}^{n}$ with $\|\phi\|, \phi \in \mathcal{C}$ defined by $\|\phi\|=\sup _{s \in[-r, 0]}|\phi(s)|, r>0$ be a real constant. If $\sigma \in \mathbb{R}$, $A>0$ and $x \in C\left([\sigma-r, \sigma+A], \mathbb{R}^{n}\right)$, then for any $t \in[\sigma, \sigma+A]$, we let $x_{t} \in \mathcal{C}$ be defined by $x_{t}(\theta)=x(t+\theta), \theta \in[-r, 0]$.

Let us recall the following known definitions. For more details, we refer the reader to [1, $2,4,5]$.

Definition 2.1 The fractional order integral of a function $f:\left[t_{0}, \infty\right) \rightarrow \mathbb{R}$ of order $\alpha \in$ $\mathbb{R}^{+}=[0,+\infty)$ is defined by

$$
I_{t_{0}}^{\alpha} f(t)=\frac{1}{\Gamma(\alpha)} \int_{t_{0}}^{t}(t-s)^{\alpha-1} f(s) d s,
$$

where $\Gamma(\cdot)$ is the gamma function.

Definition 2.2 For a function $f$ given on the interval $\left[t_{0}, \infty\right)$, the $\alpha$ order RiemannLiouville fractional derivative of $f$ is defined by

$$
D_{t_{0}}^{\alpha} f(t)=\frac{1}{\Gamma(n-\alpha)}\left(\frac{d}{d t}\right)^{n} \int_{t_{0}}^{t}(t-s)^{n-\alpha-1} f(s) d s, \quad n-1<\alpha<n, n \in \mathbb{N}
$$

where $\mathbb{N}=\{1,2,3, \ldots\}$.

Definition 2.3 For a function $f$ given on the interval $\left[t_{0}, \infty\right)$, the $\alpha$ order Caputo fractional derivative of $f$ is defined by

$$
\begin{aligned}
& { }^{c} D_{t_{0}}^{\alpha} f(t)=\frac{1}{\Gamma(n-\alpha)} \int_{t_{0}}^{t}(t-s)^{n-\alpha-1} f^{(n)}(s) d s, \quad n-1<\alpha<n, \\
& { }^{c} D_{t_{0}}^{\alpha} f(t)=f^{(n)}(t), \quad \alpha=n, n \in \mathbb{N} .
\end{aligned}
$$

Some properties of the aforementioned operators are recalled below [1]. 
Property 2.1 The following results are especially interesting:

(i) For $v>-1$, we have $D_{t_{0}}^{\alpha}\left(t-t_{0}\right)^{v}=\frac{\Gamma(1+v)}{\Gamma(1+v-\alpha)}\left(t-t_{0}\right)^{v-\alpha}$.

(ii) When $n-1<\alpha<n, n \in \mathbb{N}$, we have

$$
{ }^{c} D_{t_{0}}^{\alpha} f(t)=D_{t_{0}}^{\alpha}\left[f(t)-\sum_{i=0}^{n-1} \frac{f^{(i)}\left(t_{0}\right)\left(t-t_{0}\right)^{i}}{i !}\right] .
$$

(iii) For $\alpha \in(0,1), T \geq t_{0}$ and $f \in C\left(\left[t_{0}, T\right], \mathbb{R}^{n}\right)$, we have ${ }^{c} D_{t_{0}}^{\alpha} I_{t_{0}}^{\alpha} f(t)=f(t)$,

$$
I_{t_{0}}^{\alpha c} D_{t_{0}}^{\alpha} f(t)=f(t)-f\left(t_{0}\right) \text {. }
$$

Remark 2.1 From Property 2.1 , if ${ }^{c} D_{t_{0}}^{\alpha} f(t) \geq 0, \alpha \in(0,1)$, then for $t \geq t_{0}$, we have

(i) $f(t) \geq f\left(t_{0}\right)$.

(ii) In general, it is not true that $f(t)$ is nondecreasing in $t$.

In [21], Cruz and Hale studied a class of functional difference operators which are very useful in stability theory and the asymptotic behavior of solutions of functional differential equations of neutral type. In monograph [22], Hale et al. presented the following definitions and results of the difference operators.

For Banach spaces $X$ and $Y, \mathcal{L}(X, Y)$ is the Banach space of bounded linear mappings from $X$ to $Y$ with the operator topology. If $L \in \mathcal{L}\left(\mathcal{C}, \mathbb{R}^{n}\right)$, then the Riesz representation theorem implies that there is an $n \times n$ matrix function $\mu$ on $[-r, 0]$ of bounded variation such that

$$
L \phi=\int_{-r}^{0}[d \mu(\theta)] \phi(\theta) .
$$

Definition 2.4 Let $\Omega$ be an open subset of a metric space. We say $L: \Omega \rightarrow \mathcal{L}\left(\mathcal{C}, \mathbb{R}^{n}\right)$ has smoothness on the measure if, for any $B \in \mathbb{R}$, there is a scalar function $\gamma(\lambda, t)$ continuous for $\lambda \in \Omega, t \in \mathbb{R}, \gamma(\lambda, 0)=0$, such that if $L(\lambda) \phi=\int_{-r}^{0}[d \mu(\lambda, \theta)] \phi(\theta), \lambda \in \Omega, s>0$, then

$$
\lim _{h \rightarrow 0^{+}}\left|\int_{B+h}^{B+s}+\int_{B-s}^{B-h}[d \mu(\lambda, \theta)] \phi(\theta)\right| \leq \gamma(\lambda, s)\|\phi\| .
$$

If $B \in \mathbb{R}$ and the matrix $A(\lambda ; B, L)=\mu\left(\lambda, B^{+}\right)-\mu\left(\lambda, B^{-}\right)$is nonsingular at $\lambda=\lambda_{0}$, we say $L(\lambda)$ is atomic at $B$ at $\lambda_{0}$. If $A(\lambda ; B, L)$ is nonsingular on a set $K \subseteq \Lambda$, we say $L(\lambda)$ is atomic at $B$ on $K$.

Definition 2.5 Suppose that $\Omega \subseteq \mathbb{R} \times \mathcal{C}$ is open with elements $(t, \phi)$. A function $\mathcal{D}: \Omega \rightarrow$ $\mathbb{R}^{n}$ (not necessarily linear) is said to be atomic at $B$ on $\Omega$ if $\mathrm{D}$ is continuous together with its first and second Fréchet derivatives with respect to $\phi$; and $\mathcal{D}_{\phi}$, the derivative with respect to $\phi$, is atomic at $B$ on $\Omega$.

Remark 2.2 If $\mathcal{D}(t, \phi)$ is linear in $\phi$ and continuous in $(t, \phi) \in \mathbb{R} \times \mathcal{C}$,

$$
\mathcal{D}(t, \phi)=\int_{-r}^{0}\left[d_{\theta} \mu(t, \theta)\right] \phi(\theta)
$$

then $A(t, \phi, B)=A(t, B)$ is independent of $\phi$ and

$$
A(t, B)=\mu\left(t, B^{+}\right)-\mu\left(t, B^{-}\right) .
$$


Thus, $\mathcal{D}(t, \phi)$ is atomic at $B$ on $\mathbb{R} \times \mathcal{C}$ for all $t \in \mathbb{R}$. In particular, if $B \neq 0, B \in[-r, 0]$, $\mathcal{D}(t, \phi)=\phi(0)+F(t) \phi(B)$, then $A(t, B)=F(t)$ and $\mathcal{D}(t, \phi)$ is atomic at $B$ on $\mathbb{R} \times \mathcal{C}$ if $\operatorname{det} F(t) \neq 0$ for all $t \in \mathbb{R}$.

In the sequel, we consider the following nonlinear fractional neutral system:

$$
{ }^{c} D_{t_{0}}^{\alpha}\left[\mathcal{D}\left(t, x_{t}\right)\right]=f\left(t, x_{t}\right), \quad t>t_{0},
$$

with the initial condition

$$
x_{t_{0}}=\varphi,
$$

where $0<\alpha<1, t_{0} \in \mathbb{R}$ is a constant, $\mathcal{D}, f: \mathbb{R} \times \mathcal{C} \rightarrow \mathbb{R}^{n}$ are given continuous functions, nonlinear difference operator $\mathcal{D}$ atomic at zero. For more details about the operator $\mathcal{D}$, the reader may refer to $[21,22,24]$ and the references therein. In the sequel, we always assume that, for any given $t_{0} \in \mathbb{R}$ and a given function $\varphi \in \mathcal{C}$, there exists a unique continuous solution of (2.1), denoted by $x(t)=x\left(t, t_{0}, \varphi\right)$, such that it satisfies (2.1) for all $t>t_{0}$ and (2.2). To deal with stability, as usual, we assume that $\mathcal{D}(t, 0)=f(t, 0)=0$ so that $(2.1)$ has the zero solution.

Definition 2.6 [23] The zero solution $x=0$ of (2.1) is stable if for any $t_{0} \in \mathbb{R}$ and any $\varepsilon>0$, there exists $\delta=\delta\left(t_{0}, \varepsilon\right)$ such that any solution $x(t)=x\left(t, t_{0}, \varphi\right)$ of $(2.1)$ with initial value $\varphi$ at $t_{0},\|\varphi\|<\delta$ satisfies $|x(t)|<\varepsilon$ for $t \geq t_{0}$. It is asymptotically stable if it is stable and for any $t_{0} \in \mathbb{R}$ and any $\varepsilon>0$, there exists $\delta_{0}=\delta\left(t_{0}, \varepsilon\right), T\left(t_{0}, \varepsilon\right)>0$ such that $\|\varphi\|<\delta_{0}$ implies $|x(t)| \leq \varepsilon$ for $t \geq t_{0}+T\left(t_{0}, \varepsilon\right)$, i.e., $\lim _{t \rightarrow+\infty} x(t)=0$. It is uniformly stable if it is stable and $\delta=\delta(\varepsilon)>0$ can be chosen independently of $t_{0}$. It is uniformly asymptotically stable if it is uniformly stable and there exists $\delta_{0}>0$ for any $\eta>0$, there exists $T=T(\eta)>0$ such that $\|\varphi\|<\delta_{0}$ implies $|x(t)|<\eta$ for $t>t_{0}+T$. It is globally (uniformly) asymptotically stable if it is (uniformly) asymptotically stable and $\delta_{0}$ can be an arbitrary large, finite number.

For a nonlinear operator $\mathcal{D}$, in [24], Zhang gave the following definition.

Definition 2.7 $\mathcal{D}(t, \phi)$ is said to be uniformly stable if there exist positive constants $a, b$, $c$ such that for any $h: \mathbb{R} \rightarrow \mathbb{R}^{n}$ and any $\sigma \in \mathbb{R}, \varphi \in \mathbb{C}$, with $\mathcal{D}(\sigma, \varphi)=h(\sigma)$, the solution $x(t, \sigma, \varphi)$ of

$$
\begin{aligned}
& \mathcal{D}\left(t, x_{t}\right)=h(t), \quad t \geq \sigma, \\
& x_{\sigma}=\varphi .
\end{aligned}
$$

satisfies the following estimate:

$$
\left\|x_{t}\right\| \leq b e^{-a(t-\sigma)}\|\varphi\|+c \sup _{u \in[\sigma, t]}|h(u)|, \quad t \geq \sigma .
$$

The following lemma plays a major role in our analysis.

Lemma 2.1 Let $\mathcal{D}(t, \phi)$ be uniformly stable, $x_{t}=x_{t}(\sigma, \varphi)$ be the solution of equation (2.3). Suppose that $\alpha(t)$ is any continuous and nondecreasing function with $\alpha(0)=0$, and $\alpha(t)>0$ 
for $t>0$. Then, for small $s>0$, there is a continuous and strictly increasing function $\beta(s) \geq$ $b s+c \alpha(s), \beta(0)=0, \beta(t)>0$ for $t>0$ such that

(i) for each small $\delta>0,\|\varphi\|<\delta,\left|\mathcal{D}\left(t, x_{t}\right)\right|<\alpha(\delta), t \geq \sigma$, then

$$
\left\|x_{t}\right\| \leq \beta(\delta), \quad t \geq \sigma
$$

(ii) for each small $\delta>0, \mu>0$ and a nonnegative constant $L$, there exists $T(\mu, \delta, L)>0$ such that $\|\varphi\|<\delta$ and $\left|\mathcal{D}\left(t, x_{t}\right)\right| \leq \alpha(\mu), t \geq \sigma$ imply

$$
\left\|x_{t}\right\| \leq \beta(\mu)+L, \quad t \geq \sigma+T(\mu, \delta, L) .
$$

Proof (i) From Definition 2.5, for sufficiently small $\delta>0$ and $t \geq \sigma$, we have

$$
\begin{aligned}
\left\|x_{t}\right\| & \leq b e^{-a(t-\sigma)}\|\varphi\|+c \sup _{u \in[\sigma, t]}|h(u)| \\
& \leq b \delta+c \alpha(\delta) .
\end{aligned}
$$

Trivially, we can choose a continuous and increasing function $\beta(\delta) \geq b \delta+c \alpha(\delta), \beta(0)=0$, $\beta(t)>0$ for $t>0$, so that (2.5) holds.

(ii) For $L \geq 0$ and sufficiently small $\mu>0$, if $\|\varphi\|<\delta$ and $\left|\mathcal{D}\left(t, x_{t}\right)\right| \leq \alpha(\mu), t \geq \sigma$, then by Definition 2.5 it suffices to show that

$$
b e^{-a(t-\sigma)} \delta+c \alpha(\mu) \leq b \mu+c \alpha(\mu)+L,
$$

which implies

$$
\left\|x_{t}\right\| \leq \beta(\mu)+L, \quad t \geq \sigma+T(\mu, \delta, L) .
$$

Therefore, if we take $T(\mu, \delta, L)=\max \left\{0, \frac{1}{a} \ln \frac{b \delta}{b \mu+L}\right\}$, then (2.6) holds.

\section{Main results}

In this section, we consider the stability of nonlinear fractional neutral system (2.1). Here, we always assume that fractional neutral system (2.1) with initial condition (2.2) has a unique continuous solution $x(t)$ which depends continuously upon $t_{0}, \varphi$. By Property 2.1(iii), we can obtain that initial value problem (2.1)-(2.2) is equivalent to the integral equation

$$
\mathcal{D}\left(t, x_{t}\right)=\mathcal{D}\left(t_{0}, \varphi\right)+I_{t_{0}}^{\alpha} f\left(t, x_{t}\right), \quad t \geq t_{0}, x_{t_{0}}=\varphi .
$$

If $V: \mathbb{R} \times \mathcal{C} \rightarrow \mathbb{R}$ is continuously differentiable, we define the Caputo fractional derivative ${ }^{c} D_{t_{0}}^{\alpha} V(t, \phi)$ along the solution $x_{t}=x_{t}\left(t_{0}, \varphi\right)$ of (2.1)-(2.2) as

$$
{ }^{c} D_{t_{0}}^{\alpha} V\left(t, x_{t}\right)=\frac{1}{\Gamma(1-\alpha)} \int_{t_{0}}^{t}(t-s)^{-\alpha} V^{\prime}\left(s, x_{s}\right) d s .
$$

Now, we give the following Lyapunov-Krasovskii methods for nonlinear fractional neutral systems as counterpart to Lyapunov-Krasovskii methods for classical neutral systems proposed in [21]. 
Theorem 3.1 Suppose that $\mathcal{D}(t, \phi)$ is uniformly stable, $f$ takes closed bounded sets into bounded sets, and suppose that $u(s), v(s)$ are strictly increasing functions with $u(0)=v(0)=0$, and $w(s)$ is a continuous, nonnegative, nondecreasing function. If there exists a continuously differentiable functional $V: \mathbb{R} \times \mathcal{C} \rightarrow \mathbb{R}$ such that

$$
\begin{aligned}
& u(|\mathcal{D}(t, \phi)|) \leq V(t, \phi) \leq v(\|\phi\|), \\
& { }^{c} D_{t_{0}}^{\gamma} V\left(t, x_{t}\right) \leq-w\left(\left|\mathcal{D}\left(t, x_{t}\right)\right|\right),
\end{aligned}
$$

where $\gamma \in(0,1]$. Then the zero solution of $(2.1)$ is uniformly stable. If, in addition, $w(s)>0$ for $s>0$, then it is uniformly asymptotically stable.

Proof It is possible to choose a continuous function $\alpha(t)$ so that $\alpha(0)=0$ and $\alpha(t)>$ $u^{-1}(v(t)) \geq 0$ for small $t>0$. Then $v(t)<u(\alpha(t))$ for small $t>0$. For the above-chosen $\alpha(t)$, by Lemma 2.1, we can find a corresponding $\beta(t)$ with the desired properties. Now, for any $\varepsilon>0$, we can find a sufficiently small $\delta$ such that $\beta(\delta)<\varepsilon$. Hence, for any initial time $t_{0}$ and any initial condition $x_{t_{0}}=\varphi$ with $\|\varphi\|<\delta$, (3.2) implies

$$
{ }^{c} D_{t_{0}}^{\gamma} V\left(t, x_{t}\left(t_{0}, \varphi\right)\right) \leq 0, \quad t \geq t_{0}
$$

by Property 2.1, we have

$$
V\left(t, x_{t}\left(t_{0}, \varphi\right)\right) \leq V\left(t_{0}, \varphi\right), \quad t \geq t_{0} .
$$

By (3.2), this implies that

$$
u\left(\left|\mathcal{D}\left(t, x_{t}\left(t_{0}, \varphi\right)\right)\right|\right) \leq V\left(t, x_{t}\left(t_{0}, \varphi\right)\right) \leq V\left(t_{0}, \varphi\right) \leq v(\delta)<u(\alpha(\delta)), \quad t \geq t_{0},
$$

which implies that $\left|\mathcal{D}\left(t, x_{t}\left(t_{0}, \varphi\right)\right)\right|<\alpha(\delta)$ for $t \geq t_{0}$. Since $\mathcal{D}(t, \phi)$ is uniformly stable, Lemma 2.1 implies

$$
\left\|x_{t}\left(t_{0}, \varphi\right)\right\|<\varepsilon, \quad t \geq t_{0} .
$$

Therefore, the zero solution is uniformly stable.

To prove uniform asymptotic stability, let $\varepsilon_{0}:=\varepsilon=1$, choose $\delta_{0}:=\delta(1)>0$ corresponding to uniform stability. Then, for any $t_{0} \in \mathbb{R},\|\varphi\|<\delta_{0}$ implies

$$
\left\|x_{t}\left(t_{0}, \varphi\right)\right\|<\varepsilon_{0}, \quad\left|\mathcal{D}\left(t, x_{t}\left(t_{0}, \varphi\right)\right)\right|<\alpha\left(\delta_{0}\right), \quad t \geq t_{0} .
$$

Next, for any $\eta>0$, we wish to show that there is $T\left(\delta_{0}, \eta\right)$ such that any solution $x\left(t, t_{0}, \varphi\right)$ of (2.1) with $\|\varphi\|<\delta_{0}$ satisfies

$$
\left\|x_{t}\left(t_{0}, \varphi\right)\right\|<\eta, \quad t \geq t_{0}+T\left(\delta_{0}, \eta\right) .
$$

Suppose that (3.4) is not true, then there is a solution $x=x\left(t, t_{0}, \varphi\right)$ of (2.1) with $\|\varphi\|<\delta_{0}$ and $\left\|x_{t}\left(t_{0}, \varphi\right)\right\| \geq \delta=\delta(\eta)\left(\delta<4 b \alpha\left(\delta_{0}\right)\right)$ for $t \geq t_{0}$. Then, for any $s \geq t_{0}$, from (2.4) we have

$$
\delta \leq\left\|x_{t}\right\| \leq b e^{-a(t-s)}\left\|x_{s}\right\|+c \sup _{u \in[s, t]}\left|\mathcal{D}\left(u, x_{u}\right)\right| .
$$


Choose $N=N\left(\delta_{0}, \eta\right)$ so that $N>\max \left\{o, \frac{1}{a} \ln \frac{2 b}{\delta}\right\}$. Let $t_{1}=t_{0}+N$, from (3.5) we have

$$
\begin{aligned}
\delta \leq\left\|x_{t_{1}}\right\| & \leq b e^{-a N}\left\|x_{t_{0}}\right\|+c \sup _{u \in\left[t_{0}, t_{1}\right]}\left|\mathcal{D}\left(u, x_{u}\right)\right| \\
& \leq \frac{\delta}{2}+b \sup _{u \in\left[t_{0}, t_{1}\right]}\left|\mathcal{D}\left(u, x_{u}\right)\right| .
\end{aligned}
$$

Then we have

$$
b \sup _{u \in\left[t_{0}, t_{1}\right]}\left|\mathcal{D}\left(u, x_{u}\right)\right| \geq \frac{\delta}{2} .
$$

Therefore, there exists $\zeta_{1} \in\left[t_{0}, t_{1}\right]$ such that

$$
\left|\mathcal{D}\left(\zeta_{1}, x_{\zeta_{1}}\right)\right| \geq \frac{\delta}{2 b}
$$

Now consider a sequence of intervals $I_{k}$ with $I_{k}=\left[t_{k-1}, t_{k}\right], t_{k}=t_{0}+k N, k=1,2, \ldots$ By (3.5) and (3.6), there must be some $\zeta_{k}$ in each $I_{k}$ such that

$$
\left|\mathcal{D}\left(\zeta_{k}, x_{\zeta_{k}}\right)\right| \geq \frac{\delta}{2 b}
$$

Then there exists a sequence $\left\{\zeta_{k}\right\}_{k=1}^{\infty}, \zeta_{k} \rightarrow \infty$, as $k \rightarrow \infty$ such that

$$
\left|\mathcal{D}\left(\zeta_{k}, x_{\zeta_{k}}\right)\right| \geq \frac{\delta}{2 b}, \quad k \geq 1
$$

Without loss of generality, we may assume that $\zeta_{k+1}-\zeta_{k} \geq r$ for $k \geq 0$. It follows from equation (3.1) that

$$
\mathcal{D}\left(t, x_{t}\left(t_{0}, \varphi\right)\right)=\mathcal{D}\left(t_{0}, \varphi\right)+I_{t_{0}}^{\alpha} f\left(t, x_{t}\left(t_{0}, \varphi\right)\right), \quad t \geq t_{0},
$$

and

$$
\mathcal{D}\left(\zeta_{k}, x_{\zeta_{k}}\left(t_{0}, \varphi\right)\right)=\mathcal{D}\left(t_{0}, \varphi\right)+I_{t_{0}}^{\alpha} f\left(\zeta_{k}, x_{\zeta_{k}}\left(t_{0}, \varphi\right)\right)
$$

Then we have

$$
\mathcal{D}\left(t, x_{t}\left(t_{0}, \varphi\right)\right)=\mathcal{D}\left(\zeta_{k}, x_{\zeta_{k}}\left(t_{0}, \varphi\right)\right)+I_{t_{0}}^{\alpha} f\left(t, x_{t}\left(t_{0}, \varphi\right)\right)-I_{t_{0}}^{\alpha} f\left(\zeta_{k}, x_{\zeta_{k}}\left(t_{0}, \varphi\right)\right)
$$

Since $f$ takes bounded sets into bounded sets, there is a constant $L_{0}$ such that $f(t$, $\left.x_{t}\left(t_{0}, \varphi\right)\right) \mid \leq L_{0}$ for $t \geq t_{0},\|\varphi\| \leq \delta_{0}$. Then

$$
\left|\mathcal{D}\left(t, x_{t}\left(t_{0}, \varphi\right)\right)\right| \geq \frac{\delta}{2 b}-\frac{L_{0}\left(t-\zeta_{k}\right)^{\alpha}}{\Gamma(1+\alpha)}, \quad t \geq \zeta_{k} .
$$

Hence, for any $k \geq 1$, we have

$$
\left|\mathcal{D}\left(x_{t}\left(t_{0}, \varphi\right)\right)\right| \geq \frac{\delta}{4 b}, \quad t \in\left[\zeta_{k}, \zeta_{k}+\theta\right],
$$

where $\theta=\min \left\{r,\left[\frac{\delta \Gamma(1+\alpha)}{4 b L_{0}}\right]^{\frac{1}{\alpha}}\right\}$. 
Let

$$
\lambda=\min _{\frac{\delta}{4 b} \leq s \leq \alpha\left(\delta_{0}\right)} w(s)>0 .
$$

Then there exists some positive integer $k_{0}$ such that

$$
V\left(t_{0}, \varphi\right)<\frac{\lambda}{\Gamma(1+\gamma)}\left(\zeta_{k_{0}}-t_{0}\right)^{\gamma}
$$

By (3.2), we have

$$
{ }^{c} D_{t_{0}}^{\gamma} V\left(\zeta_{k_{0}}, x_{\zeta_{k_{0}}}\left(t_{0}, \varphi\right)\right) \leq-w\left(\left|\mathcal{D}\left(\zeta_{k_{0}}, x_{\zeta_{k_{0}}}\left(t_{0}, \varphi\right)\right)\right|\right) \leq-\lambda
$$

By (3.2), (3.7) and Property 2.1, we have

$$
0<u\left(\frac{\delta}{2 b}\right) \leq V\left(\zeta_{k_{0}}, x_{\zeta_{k_{0}}}\left(t_{0}, \varphi\right)\right) \leq V\left(t_{0}, \varphi\right)-\frac{\lambda}{\Gamma(1+\gamma)}\left(\zeta_{k_{0}}-t_{0}\right)^{\gamma}<0,
$$

which is a contradiction. Then there must be some $t^{*} \in\left[t_{0}, t_{0}+k_{0} N\right]$ such that $\| x_{t^{*}}\left(t_{0}\right.$, $\varphi) \|<\delta$. Therefore, for $\|\varphi\|<\delta_{0}$, we have

$$
\left|x\left(t, t_{0}, \varphi\right)\right|<\eta, \quad t \geq t_{0}+k_{0} N
$$

This proves the uniform asymptotic stability of the zero solution of (2.1).

Remark 3.1 From the proof of inequality (3.8), we can know that the analysis on stability of fractional differential equations is more complex than that of classical differential equations, since fractional derivatives are nonlocal and have weakly singular kernels.

Remark 3.2 If $\mathcal{D}(t, \phi)$ is linear in $\phi$ and $\alpha=\gamma=1$, Theorem 3.1 is just the same as Theorem 4.1 in [21].

Remark 3.3 If $\mathcal{D}(t, \phi)=\phi(0)$, the conclusions of Theorem 3.1 are just the same as the corresponding conclusions of Theorem in [15].

Theorem 3.2 Suppose that the assumptions in Theorem 3.1 are satisfied except replacing ${ }^{c} D_{t_{0}}^{\gamma}$ by $D_{t_{0}}^{\gamma}$, then one has the same result for uniform stability and uniform asymptotic stability.

Proof By using Property 2.1, we have

$$
{ }^{c} D_{t_{0}}^{\gamma} V\left(t, x_{t}\right)=D_{t_{0}}^{\gamma} V\left(t, x_{t}\right)-V\left(t_{0}, \varphi\right) \frac{\left(t-t_{0}\right)^{-\gamma}}{\Gamma(1-\gamma)} .
$$

Since $V\left(t_{0}, \varphi\right) \geq 0$, then ${ }^{c} D_{t_{0}}^{\gamma} V\left(t, x_{t}\right) \leq D_{t_{0}}^{\gamma} V\left(t, x_{t}\right)$. Then we can obtain the same result for uniform stability and uniform asymptotic stability.

\section{Conclusions}

In this paper, we have studied the stability of nonlinear fractional order neutral systems. We introduce the Lyapunov-Krasovskii approach for the nonlinear fractional neutral sys- 
tems, which enrich the knowledge of both the system theory and the fractional calculus. We partly extend the application of Caputo fractional systems by using LyapunovKrasovskii approach. By using Caputo and Riemann-Liouville derivatives and LyapunovKrasovskii technique, uniform stability criteria are obtained for the nonlinear fractional neutral systems. The obtained conclusions generalize the corresponding conclusions in $[15,21]$.

\section{Competing interests}

The authors declare that they have no competing interests.

\section{Authors' contributions}

All authors contributed equally in this article. They read and approved the final manuscript.

\section{Acknowledgements}

This work is supported by the National Natural Science Foundation of China (11371027), the Fundamental Research Funds for the Central Universities (2013HGXJ0226) and the Fund of Anhui University Graduate Academic Innovation Research (10117700004)

\section{Received: 6 September 2013 Accepted: 2 December 2013 Published: 27 Dec 2013}

\section{References}

1. Kilbas, AA, Srivastava, HM, Trujillo, JJ: Theory and Applications of Fractional Differential Equations. North-Holland Mathematics Studies, vol. 204. Elsevier, Amsterdam (2006)

2. Miller, KV, Ross, B: An Introduction to the Fractional Calculus and Fractional Differential Equations. Wiley, New York (1993)

3. Monje, CA, Chen, YQ, Vinagre, BM, Xue, D, Feliu, V: Fractional-Order Systems and Controls: Fundamentals and Applications. Springer, London (2010)

4. Podlubny, I: Fractional Differential Equations. Academic Press, San Diego (1999)

5. Samko, SG, Kilbas, AA, Marichev, OI: Fractional Integrals and Derivatives: Theory and Applications. Gordon and Breach, Yverdon (1993)

6. Matignon, D: Stability results for fractional differential equations with applications to control processing. In: IMACS-SMC Proceedings, Lille, France, July 1996

7. Chen, YQ, Moore, KL: Analytical stability bound for a class of delayed fractional-order dynamic systems. Nonlinear Dyn. 29, 191-200 (2002)

8. Deng, WH, Li, CP, Lü, JH: Stability analysis of linear fractional differential system with multiple time delays. Nonlinear Dyn. 48(4), 409-416 (2007)

9. Lazarevic, MP, Spasic, AM: Finite-time stability analysis of fractional order time-delay systems: Gronwall's approach. Math. Comput. Model. 49, 475-481 (2009)

10. Liu, KW, Jiang, W: Finite-time stability of linear fractional order neutral systems. Math. Appl. 24(4), 724-730 (2011)

11. Sabatier, J, Moze, M, Farges, C: LMI stability conditions for fractional order systems. Comput. Math. Appl. 59, 1594-1609 (2010)

12. Li, Y, Chen, YQ, Podlubny, I: Mittag-Leffler stability of fractional order nonlinear dynamic systems. Automatica 45(8), 1965-1969 (2009)

13. Li, Y, Chen, YQ, Podlubny, I: Stability of fractional-order nonlinear dynamic systems: Lyapunov direct method and generalized Mittag-Leffler stability. Comput. Math. Appl. 59, 1810-1821 (2010)

14. Delavari, H, Baleanu, D, Sadati, J: Stability analysis of Caputo fractional-order nonlinear systems revisited. Nonlinear Dyn. 67(4), 2433-2439 (2012)

15. Baleanu, D, Ranjbar, A, Delavari, H, Sadati, JR, Abdeljawad, T, Gejji, V: Lyapunov-Krasovskii stability theorem for fractional systems with delay. Rom. J. Phys. 56, 636-643 (2011)

16. Baleanu, D, Sadati, SJ, Ghaderi, R, Ranjbar, A, Abdeljawad, T, Jarad, F: Razumikhin stability theorem for fractional systems with delay. Abstr. Appl. Anal. 2010, Article ID 124812 (2010)

17. Sadati, SJ, Baleanu, D, Ranjbar, A, Ghaderi, R, Abdeljawad, T: Mittag-Leffler stability theorem for fractional nonlinear systems with delay. Abstr. Appl. Anal. 2010, Article ID 108651 (2010)

18. Zhang, FR, Li, CP, Chen, YQ: Asymptotical stability of nonlinear fractional differential system with Caputo derivative. Int. J. Differ. Equ. 2011, Article ID 635165 (2011)

19. Li, CP, Zhang, FR: A survey on the stability of fractional differential equations. Eur. Phys. J. Spec. Top. 193, 27-47 (2011)

20. Rivero, M, Rogosin, SV, Machado, JAT, Trujillo, JJ: Stability of fractional order systems. Math. Probl. Eng. 2013, Article ID 356215 (2013)

21. Cruz, MA, Hale, JK: Stability of functional differential equations of neutral type. J. Differ. Equ. 7, 334-355 (1970)

22. Hale, JK, Verduyn Lunel, SM: Introduction to Functional Differential Equations. Springer, New York (1993)

23. Gu, K, Kharitonov, VL, Chen, J: Stability of Time-Delay Systems. Birkhäuser, Boston (2003)

24. Zhang, SN: Unified stability theorem in RFDE and NFDE. J. Math. Anal. Appl. 150, 397-413 (1990)

10.1186/1687-1847-2013-379

Cite this article as: Liu and Jiang: Uniform stability of fractional neutral systems: a Lyapunov-Krasovskii functional approach. Advances in Difference Equations 2013, 2013:379 